\title{
VASCULAR PLANTS OF THE PETURRSON RAVINE AREA ALONG THE SOUTH SASKATCHEWAN RIVER, IN SASKATOON, SK
}

VERNON L. HARMS, \#212-115 Keevil Crescent, Saskatoon, SK S7N 4P2

\section{Introduction}

The Peturrson Ravine area in Saskatoon is a natural landscape feature frequently visited by local naturalists, and one that has been identified by the Meewasin Valley Authority (MVA) as an area of ecological significance. Found there is a diversity of habitat-associations including springfed ravines that are often calcareous to

\section{Figure 1. City of Saskatoon showing location of Peturrson Ravine}

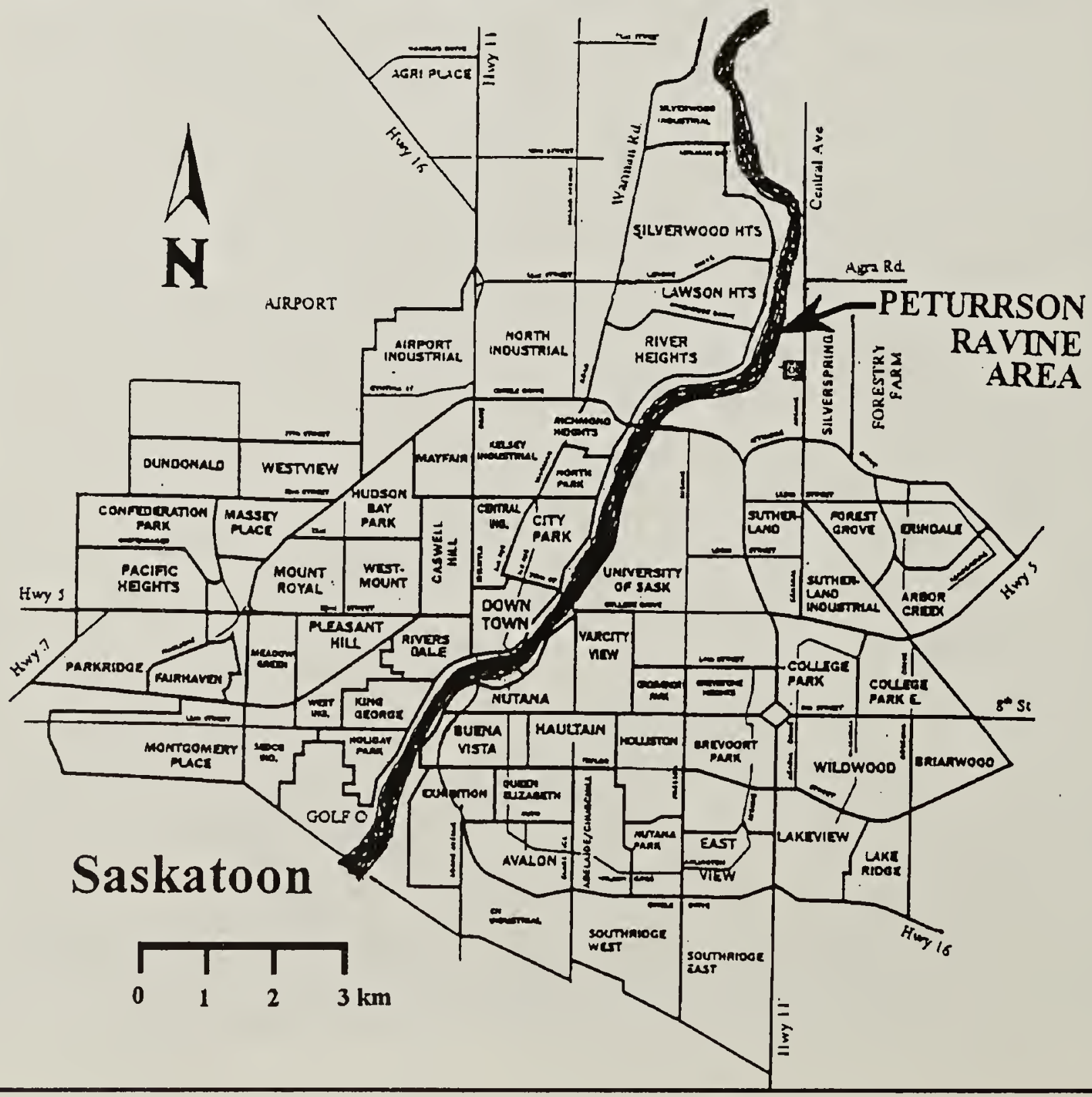


saline, wooded and open ravine and river-bluff slopes, river shores, flood plains, and upland grasslands.

This area is located in northeast Saskatoon, on the east bank of the South Saskatchewan River, north of Sutherland, west of the present Silversprings Subdivision, and just westnorthwest of the Regional Psychiatric Centre (see maps, Figures 1 and 2). It is included within the following survey coordinates: LSD 7, 9 \& 10 of Sec 11, Twp 37, Rge 5, W $3^{\text {rd }} \mathrm{M}$.

The Peturrson Ravine area is here being taken in a broad sense to include three adjacent tributary ravines respectively referred to as the "Peturrson North Ravine" (= the main
Peturrson Ravine); the "Peturrson Middle Ravine" (= the smaller ravine just south); and the "Peturrson South Ravine" (= the southernmost ravine, just west of the Regional Psychiatric Centre grounds, that was called the "Psychiatric Centre Ravine" by Lineman ${ }^{7}$ ). The north ravine extends eastward from the river to Central Avenue; its original head was cut off long ago by the construction of this road. For several years, the Saskatoon Nature Society Interpretive Centre has been located on the west side of Central Avenue just south of the ravine head, increasing public access to the area.

All three ravines are primarily springfed with groundwater-seepages along their drainages forming a complex of

Figure 2. Peturrson Ravine area (enlarged). $X$ - most calcareous marsh sites ("marl bogs")

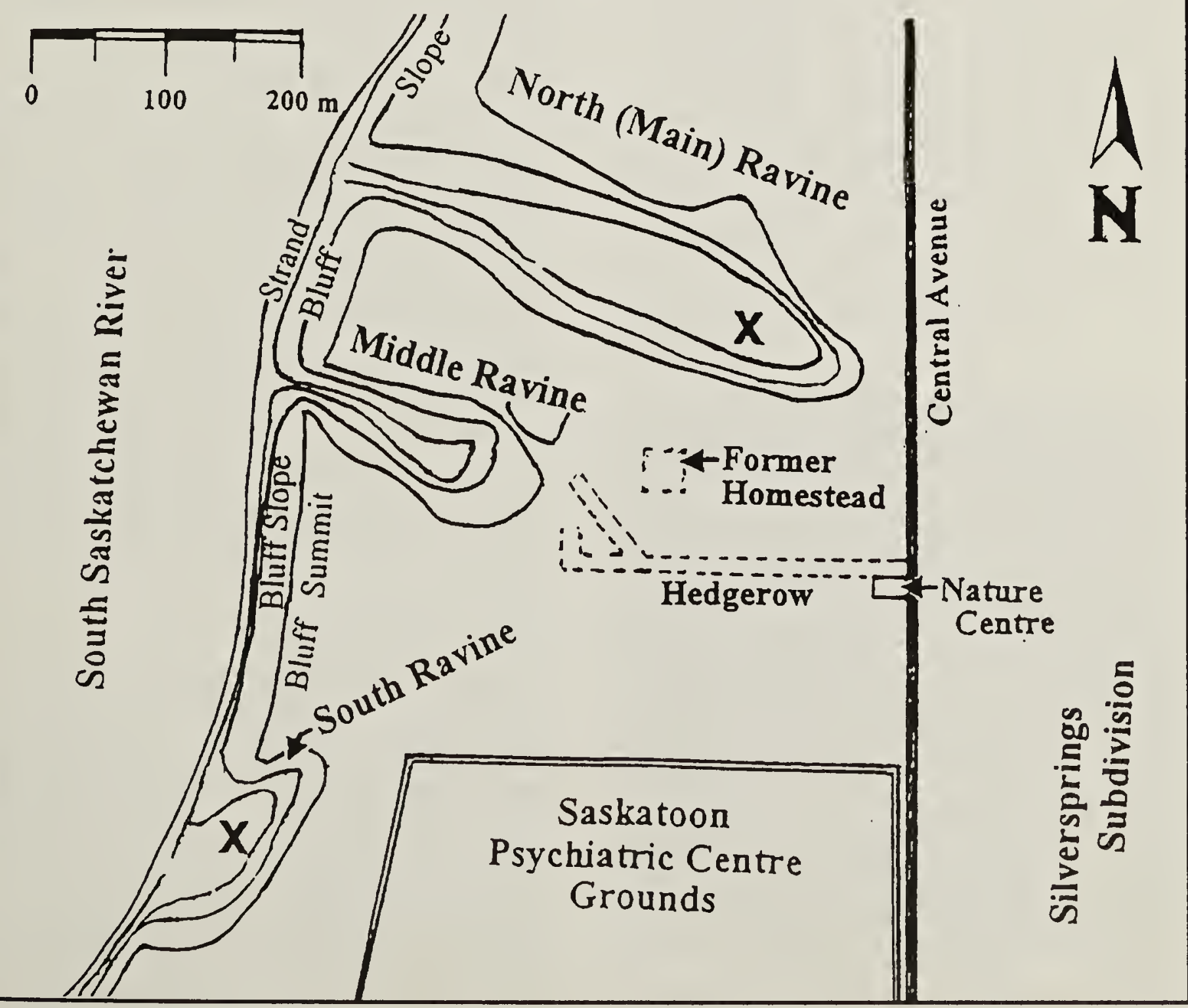


fen-like to more typical marshes. The calcareous to saline nature of some of these "seeps" is unique in the Saskatoon region, resulting in unusual botanical assemblages with the occurrence of such plants as the three provincial rarities: Red Bulrush (Blysmus rufus), Dwarf Bulrush (Trichophorum pumilum ssp. rollandii) and Marsh Felwort (Lomatogonium rotatum).$^{4}$ Together with the Riddell seepage ravine located just off Central Avenue about $1.5 \mathrm{~km}$ (about 1 mile) farther north, these constitute the Sutherland "bogs" referred to by the late W. P. Fraser ${ }^{2}$, John Hudson ${ }^{5}$ and others. Beaver-dams along the springfed ravine drainages have ponded some parts in both the north and middle ravines to create additional aquatic and marsh habitats.

While the wetland seeps and marshes constitute natural features of primary interest for the Peturrson ravine area, other relatively pristine natural plant associations also occur, especially on the floodplains, slopes, and summitbrow of the middle to south ravine, as well as on some wooded and brushy slopes of the north ravine. The ravine slopes are in part wooded by trees or taller shrubs (largely willows, Trembling Aspen, Balsam Poplar, Manitoba Maple, Green Ash, Choke-cherry, Saskatoonberry and European Buckthorn), in part short-shrubby (mainly Buckbrush, Silverberry, Wood's Wild Rose, and shorter growth of Chokecherry, Fireberry Hawthorn, Saskatoon-berry, European Buckthorn or Red-osier Dogwood), and partially open (i.e. unwooded, and dominated by forbs and grasses). Remnants of relatively pristine upland mixed-grassland exist along the bluffsummit between the middle and south ravines and just south of the southernmost one. Semi-natural upland mixed-grasslands are found south of the Nature Centre and hedgerow to the Regional Psychiatric Centre grounds.
Unfortunately, various quite disturbed stretches of landscape also exist in the overall area, particularly on the river flood plains and open slopes of the north ravine, and most of the bluff-summit uplands except for the sites listed above. An abundance of such exotic species as Smooth Brome, Kentucky Bluegrass, Crested Wheatgrass, Alfalfa, Yellow and White sweet-clover, and Perennial Sowthistle often characterize open disturbed places, and Perennial Sow-thistle and Canada Thistle are increasingly invading some marshes. The invasive exotic European Buckthorn has become common in some woods and shrublands.

The presence of disturbed sites and exotic plant species is not surprising considering that numerous human tracks and trails traverse the area, that a former homestead existed east of the middle ravine, that cattle-grazing has occurred, that gravel-excavation occurred along the north edge of the north ravine, and that the north ravine and the adjacent area across Central Avenue were used as dumping-grounds (land-fill). A major improvement resulted from a clean-up and restoration of the Peturrson Ravine area undertaken in 1991-92 with the combined efforts of the Meewasin Valley Authority, Saskatoon Nature Society, P.R. Developments Ltd., and other volunteers. A high mesh-fence was installed then to prevent further dumping. Nevertheless, scattered bits of debris may still be encountered.

\section{Acknowledgements}

Acknowledgement is given to the numerous individuals who have contributed to this plant list through the years, from the late W. P. Fraser (in the 1920 s and 1930s) until the present, with special thanks accorded to John $\mathrm{H}$. Hudson, Maurice J. Lineman and Luc Delanoy for their major floristic contributions. ${ }^{1,2,3,5,7}$ Dr. Fraser appears 
to have done the original botanizing in the area, although unfortunately he failed to pinpoint his collections beyond labeling them as being from the "Sutherland bogs". Thanks are due to Golder Associates et al ${ }^{3}$ for their plant compilations, vegetational analyses and detailed habitat-mapping of the northern part of the study area, and to Luc Delanoy ${ }^{1}$ for the more southern part. Luc also provided the author with valuable and much appreciated information and resource materials for the present article. The careful reviews of this article by John Hudson and Luc Delanoy are much appreciated.

\section{Species List}

The previous plant lists were doublechecked for accuracy and various priorlisted species have been omitted because of an apparent lack of voucher specimens to document them. Recorded in this list for the Peturrson Ravine area are 371 vascular plant taxa (= 363 species, with eight species having two varieties each in the area). Of these, 317 (or $85.5 \%$ ) are native and 54 (or $14.5 \%$ ) are exotic (i.e. introduced) plants. Thus the total area, with its variety of habitats, displays a rich floristic diversity. But with 54 exotic plants being recorded here, plus the extensive disturbed sites noted, it is clear that the area as a whole is hardly pristine. Despite the mix of disturbed to relatively natural sites, it is possible for local naturalists to observe a surprisingly high number of over 300 native vascular plant species in the overall Peturrson Ravine area. Species' lists for "natural areas" such as this are unlikely ever to be complete, but should rather be considered to represent a baseline to which observers can add more records.

The plants in the following species' list are alphabetically arranged by scientific names under their respective families, which in turn are ordered alphabetically. Common names are given in upper-case letters. Introduced (nonnative or exotic) plants are indicated by an asterisk $\left({ }^{*}\right)$, with all plants lacking an asterisk being considered native. Names in this listing are updated (modernized) to include most of the name changes associated with the numerous recent taxonomic revisions by authors, especially for the continuing Flora of North America Project and by the Biota of North America Program (BONAP) ${ }^{6}$, excepting when contradicted by the author's personal knowledge and preferences. Important synonyms are given in square brackets to interconnect (i.e. "peg-down") the modern names with those previously most often used and better known. Newer changes at the generic-level (but not changed species' epithets within a genus) are crossreferenced by bracketed entries in the list. Authorships, valuable for referencing them, are provided for scientific names. The abbreviation "auct." indicates a name previously used by authors but not considered a true synonym. The plant records are mostly documented by specimen-vouchers filed in the W.P. Fraser Herbarium (SASK), University of Saskatchewan, Saskatoon, SK, although some sight-records are documented by vouchers from nearby sites.

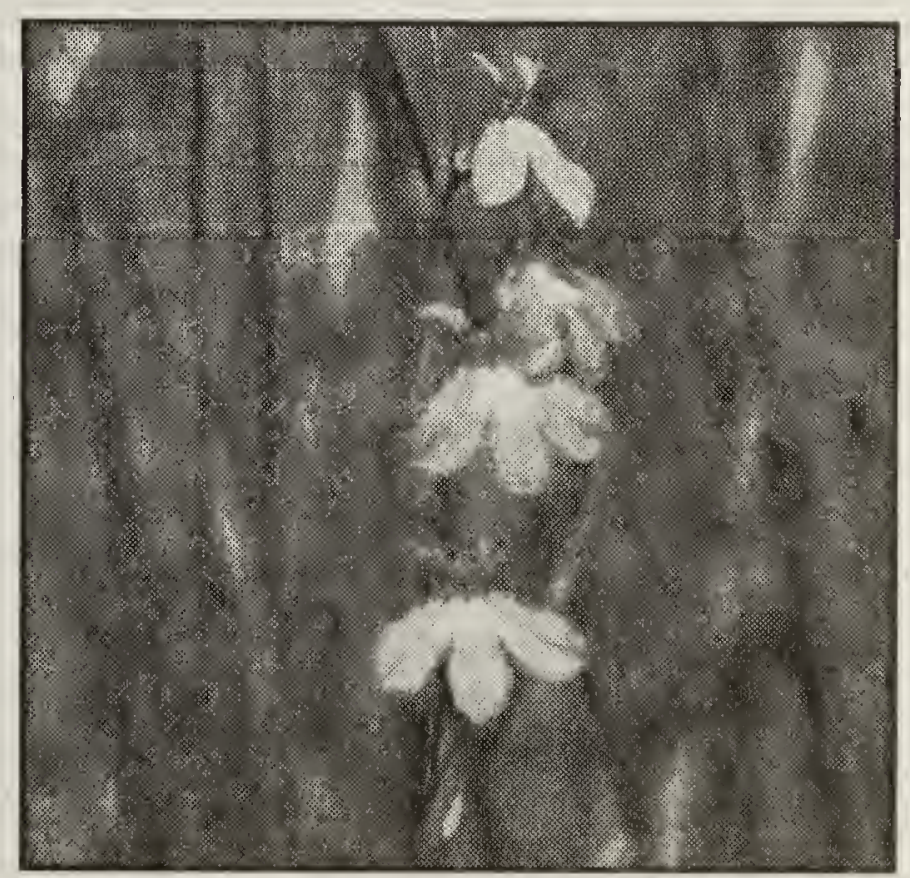

Kalm's Lobelia

Anna Leighton 
ACERACEAE (Maple Family):

-Acer negundo L.var. interius (Britt.) Sarg. - MANITOBA MAPLE; BOX-ELDER.

ALISMATACEAE (Water-plantain Family):

-Alisma triviale Pursh [A. plantago-aquatica L. var. americanum Schult. \&

Schult.] - COMMON WATER-PLANTAIN.

AMARANTHACEAE (Pigweed Family):

-Amaranthus blitoides S.Wats. * [A. graecizans auct. non L.] - PROSTRATE AMARANTH.

-Amaranthus retroflexus L. var. retroflexus * - REDROOT PIGWEED.

ANACARDIACEAE (Sumac Family):

[Rhus radicans (=Toxicodendron rydbergii)].

-Toxicodendron rydbergii (Small) Greene [Rhus radicans L. var. rydbergii Small] -POISON IVY.

\section{APIACEAE (=UMBELLIFERAE) (Parsley Family):}

- Cicuta maculata L. var. angustifolium Hook. [C. douglasii (DC.) C. \& R.] WESTERN WATER-HEMLOCK.

-Heracleum maximum Bartr. [H. lanatum Michx.] - COW PARSNIP.

-Lomatium foeniculaceum (Nutt.) C.\& R. ssp. foeniculaceum [L. villosum Raf.] HAIRY, HAIRY-FRUITED, or FENNEL-LEAVED PARSLEY.

-Lomatium macrocarpum (Nutt.) C.\& R. - LARGE-FRUITED WILD-PARSLEY or BISCUITROOT.

-Sanicula marilandica L. - BLACK SNAKEROOT.

-Sium suave Walt. - WATER PARSNIP.

-Zizia aptera (Gray) Fern. - HEART-LEAVED MEADOW-PARSNIP or

ALEXANDERS.

\section{APOCYNACEAE (Dogbane Family):}

-Apocynum androsaemifolium L. - SPREADING DOGBANE.

-Apocynum cannabinum L. var. hypericifolium Gray [A. sibiricum Jacq.] INDIAN HEMP; HEMP DOGBANE.

ARALIACEAE (Ginseng Family):

-Aralia nudicaulis L. - WILD SARSAPARILLA.

ASTERACEAE (=COMPOSITAE) (Sunflower Family):

-Achillea millefolium L. var. lanulosa (Nutt.) Piper - WOOLLY YARROW.

- Achillea sibirica Ledeb. - SIBERIAN or MANY-FLOWERED YARROW.

-Agoseris glauca (Pursh) Raf. - SMOOTH AGOSERIS; FALSE DANDELION.

- Antennaria parvifolia Nutt. — LOW or SMALL-LEAVED PUSSYTOES.

-Artemisia biennis Willd. var. biennis - BIENNIAL WORMWOOD.

- Artemisia campestris L. - TALL PLAINS SAGEWORT or WORMWOOD.

-Artemisia cana Pursh - HOARY SAGEBRUSH.

-Artemisia dracunculus L. - LINEAR-LEAVED WORMWOOD; DRAGONWORT.

- Artemisia frigida Willd. - PASTURE SAGE.

-Artemisia ludoviciana Nutt. - PRAIRIE or WHITE SAGE.

[Aster borealis (=Symphyotrichum boreale)]. 
[Aster brachyactis (=Symphyotrichum ciliatum)].

[Aster ciliolatus (=Symphyotrichum ciliolatum)].

[Aster ericoides var. pansus (=Symphyotrichum ericoides var. pansum)].

[Aster falcatus (=Symphyotrichum falcatum)].

[Aster hesperius (=Symphyotrichum lanceolatum var. hesperium)].

[Aster laevis var. geyeri (=Symphyotrichum laeve var. geyeri)].

[Chrysopsis villosa (=Heterotheca villosa)].

- Cirsium arvense (L.) Scop. * - CANADA or CREEPING THISTLE.

- Cirsium flodmanii (Rydb.) Arthur - FLODMAN'S THISTLE.

-Conyza canadensis (L.) Cronq. [Erigeron canadensis L.] - HORSEWEED; CANADA FLEABANE.

-Crepis runcinata (James) T. \& G. - SCAPOSE or DANDELION

HAWKSBEARD.

-Crepis tectorum L. * NARROW-LEAVED HAWKSBEARD.

-Erigeron asper Nutt. — ROUGH FLEABANE.

-Erigeron caespitosus Nutt. - TUFTED FLEABANE.

[Erigeron canadensis (=Conyza canadensis)].

-Erigeron lonchophyllus Hook. - HIRSUTE or SPEAR-LEAVED FLEABANE.

-Erigeron philadelphicus L. - PHILADELPHIA FLEABANE.

-Erigeron strigosus Muhl. [E. ramosus (Walt.) BSP.; E. annuus auct. non (L.)

Pers.] - DAISY or PRAIRIE FLEABANE; WHITE-TOP.

-Euthamia graminifolia (L.) Nutt. [Solidago graminifolia (L.) Salisb.] — GRASS-

LEAVED or FLAT-TOP GOLDENROD.

- Gaillardia aristata Pursh - BLANKET-FLOWER; GREAT-FLOWERED

GAILLARDIA; BROWN-EYED SUSAN.

- Grindelia squarrosa (Pursh) Dunal - CURLY-CUP GUMWEED.

-Gutierrezia sarothrae (Pursh) Britt. \& Rusby - COMMON BROOMWEED;

BROOM-SNAKEWEED.

[Haplopappus nuttallii (=Machaeranthera grindelioides)].

[Haplopappus spinulosus (=Machaeranthera pinnatifida)].

- Helenium autumnale L. - COMMON or FALL SNEEZEWEED.

-Helianthus nuttallii T.\& G. ssp. nuttallii [H. subtuberosus (Britt.) Britt.] -

NUTTALL'S, CLUSTERED, COMMON or TALL SUNFLOWER.

- Helianthus subrhomboideus Rydb - SUBRHOMBIC or BEAUTIFUL

SUNFLOWER. [H. laetiflorus auct. non Pers.]

-Heterotheca villosa (Pursh) Shinners [Chrysopsis villosa Pursh] - HAIRY

GOLDEN ASTER.

- Hieracium umbellatum L. - NARROW-LEAVED HAWKWEED.

-Iva axillaris Pursh - POVERTY-WEED; DEER-ROOT.

-Lactuca pulchella Pursh [L. tatarica (L.) Mey. var. pulchella (Pursh) Breit.] COMMON BLUE LETTUCE.

-Lactuca serriola L. - LOBED PRICKLY LETTUCE. [L. scariola L.].

-Liatris ligulistylis (A.Nels.) Schum. - MEADOW BLAZING-STAR.

- Liatris punctata Hook. - DOTTED or PUNCTATE BLAZING-STAR.

-Lygodesmia juncea (Pursh) D.Don. - RUSH SKELETON-WEED.

-Machaeranthera grindelioides (Nutt.) Shinners - TOOTHED IRONPLANT.

[Haplopappus nuttallii T.\&G.].

-Machaeranthera pinnatifida (Hook.) Shin. [Haplopappus spinulosus (Pursh) DC.] - SPINY or CUTLEAF IRON PLANT.

-Oligoneuron rigidum (L.) Small var. humile (Porter) Nesom [Solidago rigida L. 
var. humilis Porter] - STIFF, RIGID, or CORYMBOSE GOLDENROD.

-Packera cana (Hook.) Weber \& Love [Senecio canus Hook.] - SILVERY GROUNDSEL.

-Ratibida columnifera (Nutt.) Woot.\& Standl. — LONG-HEADED or YELLOW PRAIRIE CONEFLOWER.

[Senecio canus (=Packera cana)].

-Senecio integerrimus Nutt. var. integerrimus - ENTIRE-LEAVED GROUNDSEL or RAGWORT.

-Solidago canadensis L. var. canadensis - CANADA or GRACEFUL GOLDENROD.

-Solidago canadensis L. var. gilvocanescens Rydb. [S. pruinosa Greene] CANADA or CANESCENT GOLDENROD.

-Solidago gigantea Ait. — LATE or GIANT GOLDENROD.

-Solidago graminifolia (=Euthamia graminifolia).

-Solidago missouriensis Nutt. — LOW or PRAIRIE GOLDENROD.

-Solidago mollis Bartl. var. mollis - VELVETY or ASHY GOLDENROD.

-Solidago rigida var. humilis (=Oligoneuron rigidum var. humile).

-Solidago simplex Kunth var. simplex [S. spathulata DC. var. neomexicana (Gray) Cronq.] — MOUNTAIN GOLDENROD.

-Sonchus arvense L. ssp. uliginosus (Bieb.) Nyman * [S. a. var. glabrescens Guenth., Grab. \& Wimm.] - PERENNIAL or FIELD SOW-THISTLE.

-Sonchus asper (L.) Hill * - PRICKLY or SPINY-LEAVED ANNUAL SOWTHISTLE.

-Symphyotrichum boreale (T.\& G.) A.\& D. Löve [Aster borealis (T.\&G.) Prov.; A. junciformis Rydb.] - NORTHERN MARSH or BOG ASTER.

-Symphyotrichum ciliatum (Ledeb.) Nesom [Aster brachyactis Blake] RAYLESS ASTER.

-Symphyotrichum ciliolatum (Lindl.) A.\& D. Löve [Aster ciliolatus Lindl.] LINDLEY'S BLUE ASTER.

-Symphyotrichum ericoides (L.) Nesom var. pansum (Blake) Nesom [Aster ericoides L. var. pansus (Blake) Boivin] - TUFTED WHITE PRAIRIE ASTER; HEATH or MULTI-FLOWERED ASTER.

-Symphyotrichum falcatum (Lindl.) Nesom var. commutatum (T.\&G.) Nesom [Aster falcatus Lindl. var. commutatus (T.\&G.) A.G.Jones] - CREEPING WHITE PRAIRIE or HEATH ASTER.

-Symphyotrichum laeve (L.) A.\& D.Love var. geyeri (Gray) Nesom [Aster laevis L. var. geyeri Gray] — SMOOTH BLUE ASTER.

-Symphyotrichum lanceolatum (Willd.) Nesom var. hesperium (Gray) Nesom [Aster hesperius Gray] — WESTERN WILLOW ASTER; RUSH ASTER.

-Tanacetum vulgare L. * - COMMON TANSY.

-Taraxacum officinale Weber * - COMMON DANDELION.

-Tragopogon dubius Scop. * - YELLOW GOAT'S-BEARD.

-Xanthium strumarium L. - COMMON COCKLEBUR.

BETULACEAE (Birch Family):

-Betula occidentalis Hook. [B. fontinalis Sarg.] - RIVER or WATER BIRCH. -Betula pumila L. var. glandulifera Regel - SHRUB or SWAMP BIRCH. -Corylus cornuta Marsh. - BEAKED HAZELNUT. 
BORAGINACEAE (Borage Family):

-Hackelia deflexa (Wahl.) Opiz. var. americana (Gray) Fern.\& I.M.Johnston [H. americana (Gray) Fern.] - NODDING STICKSEED.

- Hackelia floribunda (Lehm.) I.M.Johnston - LARGE-FLOWERED STICKSEED. -Lappula squarrosa (Retz.) Dum. * [L. echinata Gilib.; L. myosotis Moench] BLUEBUR; STICKSEED.

-Lithospermum incisum Lehm. - NARROW-LEAVED PUCCOON..

BRASSICACEAE (=CRUCIFERAE) (Mustard Family):

- Arabis divaricarpa A.Nels. - PURPLE ROCK-CRESS.

-Arabis glabra (L.) Bernh. *? - TOWER MUSTARD.

-Arabis hirsuta (L.) Scop. var. pycnocarpa (Hopk.) Roll. - HIRSUTE or HAIRY ROCK-CRESS.

-Arabis holboellii Hornem. var. retrofracta (Grah.) Rydb. - REFLEXED ROCKCRESS.

-Descurainia sophia (L.) Webb * - FLIXWEED; FLIXWEED TANSY-MUSTARD. -Erysimum asperum (Nels.) DC. - WESTERN WALLFLOWER or PRAIRIE ROCKET.

-Erysimum inconspicuum (S.Wats.) MacM. - SMALL-FLOWERED ROCKET or WALLFLOWER.

-Hesperis matronalis L. * - DAME'S-ROCKET; SWEET ROCKET.

-Lepidium densiflorum Schrad. - COMMON PEPPERGRASS; PRAIRIE PEPPERWEED.

-Lesquerella arenosa (Richards.) Rydb. [L. ludoviciana (Nutt.) S.Wats. var. arenosa (Richards.) S.Wats.] - SAND BLADDERPOD.

-Rorippa palustris (L.) Bess. - MARSH YELLOW-CRESS

-Sisymbrium loeselii L. * - TALL HEDGE MUSTARD.

-Thlaspi arvense L.* — FIELD PENNY-CRESS; STINKWEED.

CAMPANULACEAE (Bluebell Family):

-Campanula rotundifolia L. - HAREBELL; COMMON BLUEBELL.

CAPRIFOLIACEAE (Honeysuckle Family):

-Lonicera dioica L. var. glaucescens (Rydb.) Butt. - TWINING or LIMBER HONEYSUCKLE.

-Lonicera tatarica L. * - TARTARIAN HONEYSUCKLE.

-Sambucus racemosa L. ssp. pubens (Michx.) House * - RED ELDERBERRY. -Symphoricarpos albus (L.) Blake - NORTHERN or COMMON SNOWBERRY. -Symphoricarpos occidentalis Hook. — WESTERN SNOWBERRY; BUCKBRUSH.

-Viburnum edule (Michx.) Raf. - LOW BUSH-CRANBERRY; MOOSE-BERRY; PEMBINA.

- Viburnum opulus L. var. americanum Ait. - HIGH BUSH-CRANBERRY; PEMBINA.

CARYOPHYLLACEAE (Pink Family):

[Arenaria lateriflora (=Moehringia lateriflora)].

-Cerastium arvense L. - FIELD or PRAIRIE CHICKWEED.

-Gypsophila paniculata L. * - TALL BABY'S-BREATH.

-Moehringia lateriflora (L.) Fenzl [Arenaria lateriflora L.] — GROVE or BLUNT- 
LEAVED SANDWORT.

-Silene cserei Baumg. - SMOOTH CATCHFLY.

-Silene drummondii Hook. var. drummondii [Lychnis drummondii (Hook.)

S.Wats.] - DRUMMOND'S COCKLE or CATCH-FLY.

-Silene menziesii Hook. - MENZIES' CATCHFLY.

-Stellaria crassifolia Ehrh. - FLESHY STARWORT.

CHENOPODIACEAE (Goosefoot Family):

-Atriplex gardneri (Moq.) D. Dietr. [A. nuttallii auct. non S.Wats.] - GARDNER'S or NUTTALL'S SALTBUSH or ATRIPLEX.

-Atriplex hortensis L. * - GARDEN ORACHE.

-Atriplex patula L. - SPREADING SALTBUSH or ORACHE.

-Axyris amaranthoides L.* — RUSSIAN PIGWEED.

-Chenopodium album L. * - LAMB'S-QUARTERS; WHITE PIGWEED.

-Chenopodium capitatum (L.) Ambr. — STRAWBERRY BLITE; STRAWBERRY

SPINACH.

-Chenopodium pratericola Rydb. [C. leptophyllum auct. non (Moq.) Nutt.] NARROW-LEAVED or DESERT GOOSEFOOT.

-Chenopodium salinum Standl. [C. glaucum L. var. salinum (Standl.) Boiv.] SALINE, OAK-LEAF, or SMOOTH GOOSEFOOT..

-Corispermum pacificum Mosyakin [C. hyssopifolium auct. non L.] - BUGSEED. -Kochia scoparia (L.) Schrad. * - KOCHIA; BURNING-BUSH; SUMMER-

CYPRESS;

-Monolepis nuttalliana (Schultes) Greene - SPEAR-LEAVED GOOSEFOOT; NUTTALL'S POVERTY-WEED.

-Salsola tragus L. * [S. kali L. ssp. tenuifolia Moq.; S. pestifer A.Nels.] RUSSIAN THISTLE; PRAIRIE TUMBLEWEED.

\section{CORNACEAE (Dogwood Family):}

-Cornus sericea L. ssp. stolonifera (Michx.) Fosb. [C. alba auct. non L.] - REDOSIER DOGWOOD.

CUPRESSACEAE (Cypress Family):

-Juniperus communis L. var. montana Ait. - COMMON, LOW, or GROUND JUNIPER.

-Juniperus horizontalis Moench - CREEPING JUNIPER or SAVIN.

CUSCUTACEAE (Dodder Family):

-Cuscuta gronovii Willd. - COMMON DODDER (parasitic on Canada Goldenrod).

\section{CYPERACEAE (Sedge Family):}

-Blysmus rufus (Huds.) Link [Scirpus rufus (Huds.) Schrad.] - RED BULRUSH.

SK Rare: THR.

-Carex aquatilis Wahl. - WATER SEDGE.

- Carex atherodes Spreng. - AWNED SEDGE.

-Carex duriuscula C.A. Mey. [C. eleocharis Bailey; C. stenophylla Wahl. ssp. eleocharis (Bailey) Hultén] - LOW SEDGE.

-Carex filifolia Nutt. - THREAD-LEAVED SEDGE.

-Carex foenea Willd. [C. siccata Dewey] - HAY or DRY-SPIKE SEDGE. 
-Carex inops Bailey ssp. heliophila (Mack.) Crins [C. pensylvanica Lam. var. digyna Boeckl.] - SUN-LOVING SEDGE.

- Carex lasiocarpa Ehrh. var. americana Fern. [C. lanuginosa auct. non Michx.] WOOLLY SEDGE.

-Carex obtusata Lilj. - BLUNT SEDGE.

- Carex parryana Dewey - PARRY'S SEDGE.

- Carex peckii Howe - PECK'S SEDGE.

- Carex praegracilis Boott - GRACEFUL SEDGE; CLUSTERED FIELD SEDGE. - Carex rossii Boott - ROSS' SEDGE.

-Carex sprengellii Dewey ex Spreng. - SPRENGEL'S or LONG-BEAKED

SEDGE.

- Carex utriculata Boott - BEAKED SEDGE. [C. rostrata auct. non Stokes].

-Eleocharis acicularis (L.) R. \& S. - NEEDLE SPIKE-RUSH.

-Eleocharis palustris (L.) R.\& S. - CREEPING, COMMON or PALE SPIKERUSH..

-Eleocharis quinqueflora (F.Hartm.) Schwarz - FEW-FLOWERED SPIKERUSH..

-Schoenoplectus acutus (Muhl ex Bigel.) A.\& D.Love [Scirpus acutus Muhl. ex Bigel.] - HARDSTEM or VISCID GREAT BULRUSH.

-Schoenoplectus pungens (Vahl.) Palla [Scirpus pungens Vahl.; S. americanus auct. non Pers.] - THREE-SQUARE or AMERICAN BULRUSH.

-Schoenoplectus tabernaemontani (Gmel.) Palla [Scirpus validus Vahl.; S. americanus auct. non Pers.] - COMMON or SOFT-STEM GREAT BULRUSH. [Scirpus acutus (=Schoenoplectus acutus)].

-Scirpus microcarpus J.\& K. Presl. - SMALL-FRUITED BULRUSH.

[Scirpus pumilus ssp. rollandii (=Trichophorum pumilum ssp. rollandii)].

[Scirpus pungens (=Schoenoplectus pungens)].

[Scirpus rufus (=Blysmus rufus)].

[Scirpus validus (=Schoenoplectus tabernaemontani)].

-Trichophorum pumilum (Vahl.) Schinz. \& Thell. ssp. rollandii (Fern.) Taylor \& MacBr. [Scirpus pumilus Vahl. ssp. rollandii (Fern.) Raym.] - DWARF BULRUSH. SK Rare: THR.

\section{ELAEAGNACEAE (Oleaster Family):}

-Elaeagnus angustifolia L. * - RUSSIAN OLIVE.

-Elaeagnus commutata Bernh. ex Rydb. - SILVERBERRY; WOLF-WILLOW. -Shepherdia argentea (Pursh) Nutt. - THORNY or SILVERY BUFFALO-BERRY. -Shepherdia canadensis (L.) Nutt. - CANADA, LOW or RUSSET BUFFALOBERRY; SOAPBERRY.

\section{EQUISETACEAE (Horsetail Family):}

-Equisetum arvense L. - COMMON or FIELD HORSETAIL.

-Equisetum hyemale L. ssp. affine (Engelm.) Calder \& Taylor - COMMON or

TALL SCOURING-RUSH.

-Equisetum laevigatum A.Br. - SMOOTH SCOURING-RUSH.

-Equisetum palustre L. - MARSH HORSETAIL.

\section{ERICACEAE (Heath Family):}

-Arctostaphylos uva-ursi (L.) Spreng. - COMMON BEARBERRY; KINNIKINICK. 
EUPHORBIACEAE (Spurge Family):

-Chamaesyce glyptosperma (Engelm.) Small [=Euphorbia glyptosperma Engelm.] 一. RIB-SEED, RIDGE-SEEDED or PROSTRATE SPURGE.

-Chamaesyce serpyllifolia (Pers.) Small ssp. serpyllifolia [Euphorbia serpyllifolia Pers.] - THYME-LEAVED SPURGE or SAND-MAT.

[Euphorbia glyptosperma (=Chamaesyce glyptosperma)].

[Euphorbia serpyllifolia (=Chamaesyce serpyllifolia)].

\section{FABACEAE (=LEGUMINOSAE) (Pea Family):}

-Astragalus adsurgens Pall. ssp. robustior (Hook.) Welsh [A. striatus Nutt.] ASCENDING PURPLE, GROOVED or PRAIRIE MILK-VETCH.

-Astragalus agrestis Dougl. ex G.Don [A. goniatus Nutt.; A. dasyglottis Fisch. ex DC.; - A. danicus Retz. var. dasyglottis (Fisch. ex DC.) Boiv.] - PURPLE or FIELD MILK-VETCH; COCK'S-HEAD.

-Astragalus bisulcatus (Hook.) Gray - TWO-GROOVED MILK-VETCH. -Astragalus flexuosus (Hook.) Dougl. ex G.Don - SLENDER or FLEXILE MILKVETCH.

-Astragalus gilviflorus Sheldon [A. triphyllus Pursh] - CUSHION or PLAINS MILK-VETCH.

-Astragalus missouriensis Nutt. - MISSOURI MILK-VETCH.

-Astragalus pectinatus (Hook.) Dougl. ex G.Don - NARROW-LEAVED or TINELEAVED MILK-VETCH.

-Astragalus tenellus Pursh - LOOSE-FLOWERED or PULSE MILK-VETCH. -Caragana arborescens Lam. * - SIBERIAN PEA-SHRUB; COMMON CARAGANA.

-Dalea candida Willd. var. candida [Petalostemon candidum (Willd.) Michx.] WHITE PRAIRIE-CLOVER.

-Dalea purpurea Vent. var. purpurea [Petalostemon purpureum (Vent.) Rydb] PURPLE PRAIRIE-CLOVER.

- Glycyrrhiza lepidota Pursh - AMERICAN or WILD LICORICE.

- Hedysarum alpinum L. var. americanum Michx. - AMERICAN or ALPINE HEDYSARUM or SWEET-VETCH.

-Lathyrus ochroleucus Hook. - CREAM-COLORED VETCHLING or PEA-VINE. -Medicago falcata L. * [M. sativa L. ssp. falcata (L.) Arc. - YELLOW ALFALFA or LUCERNE.

-Medicago lupulina L. * - BLACK MEDICK.

-Medicago sativa L. * - ALFALFA; LUCERNE.

-Melilotus alba Medic. ${ }^{*}$ - WHITE SWEET-CLOVER.

-Melilotus officinalis (L.) Lam. * - YELLOW SWEET-CLOVER.

-Oxytropis monticola Gray ssp. monticola [Oxytropis campestris (L.) DC. var. gracilis (A. Nels.) Barn.] - LATE YELLOW or SLENDER LOCOWEED.

-Oxytropis sericea Nutt. var. spicata (Hook.) Barn. - EARLY YELLOW LOCOWEED.

-Pediomelum argophyllum (Pursh) Grimes [Psoralea argophylla Pursh] SILVER-LEAF PSORALEA or SCURF-PEA.

-Pediomelum esculentum (Pursh) Rydb. [Psoralea esculenta Pursh] - INDIAN BREADROOT; BREADROOT SCURF-PEA; PRAIRIE TURNIP.

[Petalostemon candidum (=Dalea candida)].

[Petalostemon purpureum (=Dalea purpurea)].

[Psoralea argophylla (=Pediomelum argophyllum)]. 
[Psoralea esculenta (=Pediomelum esculentum)].

[Psoralea lanceolata (=Psoralidium lanceolatum)].

-Psoralidium lanceolatum (Pursh) Rydb. [Psoralea lanceolata Pursh] - LANCELEAVED PSORALEA or SCURF-PEA.

- Thermopsis rhombifolia (Nutt. ex Pursh) Nutt. ex Richards. - GOLDEN BEAN; YELLOW BUFFALO BEAN.

- Vicia americana Muhl. ex Willd. ssp. americana - AMERICAN VETCH.

- Vicia americana Muhl. ex Willd. ssp. minor (Hook.) C.R. Gunn [V. sparsifolia Nutt. ex T.\& G.] — NARROW-LEAVED AMERICAN VETCH.

- Vicia cracca L. ssp. cracca * - TUFTED or BIRD VETCH.

FUMARIACEAE (Fumitory Family):

-Corydalis aurea Willd. - GOLDEN CORYDALIS.

GENTIANACEAE (Gentian Family):

[Gentiana crinita ssp. macounii (=Gentianopsis macounii)].

-Gentianopsis macounii (Holm) IItis [Gentiana macounii Holm.; Gentianella crinita (Froel.) G.Don ssp. macounii (Holm.) Gillett] — PRAIRIE or MACOUN'S FRINGED GENTIAN.

-Lomatogonium rotatum (L.) Fries ex Fern. - MARSH FELWORT. SK Rare: VUL.

GROSSULARIACEAE (Currant Family):

-Ribes americanum P.Mill. - WILD or AMERICAN BLACK CURRANT. -Ribes oxyacanthoides L. ssp. oxyacanthoides - NORTHERN or CANADA GOOSE-BERRY.

IRIDACEAE (Iris Family):

-Sisyrinchium montanum Greene - COMMON BLUE-EYED GRASS.

JUNCACEAE (Rush Family):

-Juncus alpinoarticulatus Chaix ssp. nodulosus (Wahl.) Hämet-Ahti - ALPINE RUSH. [J. alpinus Vill. var. rariflorus Hartm.].

-Juncus balticus Willd. - BALTIC or WIRE RUSH.

-Juncus bufonius L. - TOAD or SEASIDE RUSH.

JUNCAGINACEAE (Arrow-grass Family):

-Triglochin maritima L. - SEASIDE or SHORE ARROW-GRASS.

-Triglochin palustre L. - MARSH or SLENDER ARROW-GRASS.

LAMIACEAE (=LABIATAE) (Mint Family):

-Lycopus americanus Muhl. ex Bart. - CUT-LEAVED or AMERICAN WATERHOREHOUND.

-Lycopus asper Greene - ROUGH or WESTERN WATER-HOREHOUND.

-Mentha arvensis L. - WILD or FIELD MINT.

LILIACEAE (Lily Family):

-Allium textile A.Nels. \& Macbr. - PRAIRIE ONION. -Asparagus officinalis L.* - GARDEN ASPARAGUS.

-Disporum trachycarpum (S.Wats.) Benth. \& Hook. - FAIRYBELLS. 
-Maianthemum stellatum (L.) Link. [Smilacina stellata (L.) Desf.] - STARFLOWERED SOLOMON'S-SEAL.

[Smilacina stellata (=Maianthemum stellatum)].

-Zygadenus elegans Pursh - SMOOTH or WHITE CAMAS.

LINACEAE (Flax Family):

-Linum lewisii Pursh [L. perenne L. ssp. lewisii (Pursh) Hult.] - LEWIS' WILD BLUE FLAX.

-Linum rigidum Pursh - YELLOW FLAX.

LOBELIACEAE (Lobelia Family):

-Lobelia kalmii L. - KALM'S, BROOK or BOG LOBELIA.

MALVACEAE (Mallow Family):

-Malva rotundifolia L. ${ }^{*}$ - ROUND-LEAVED or COMMON MALLOW. [M. pusilla Sm.].

-Sphaeralcea coccinea (Nutt.) Rydb. [Malvastrum coccineum (Nutt.) Gray] SCARLET MALLOW.

NYCTAGINACEAE ( Four-o'clock Family):

-Mirabilis albida (Walt.) Heim. [M. hirsuta (Pursh) MacM.] - HAIRY

UMBRELLAWORT or FOUR-O'CLOCK.

OLEACEAE (Olive Family):

- Fraxinus pennsylvanica Marsh. - GREEN ASH.

-Syringa vulgaris $L .{ }^{*}$ - COMMON LILAC.

ONAGRACEAE (=OENOTHERACEAE) (Evening-Primrose Family):

-Epilobium ciliatum Raf. [E. glandulosum Lehm. var. adenocaulon (Haussk.)

Fern.] - NORTHERN WILLOW-HERB.

-Epilobium leptophyllum Raf. - NARROW-LEAVED or BOG WILLOW-HERB. -Epilobium palustre L. [E. lineare Muhl.] - MARSH WILLOW-HERB.

- Gaura coccinea Nutt. ex Pursh - SCARLET GAURA or BUTTERFLY-PLANT. -Oenothera biennis L. - YELLOW or COMMON EVENING-PRIMROSE.

ORCHIDACEAE (Orchid Family):

[Habenaria hyperborea (=Platanthera hyperborea)].

-Platanthera hyperborea (L.) Lindl. [Habenaria hyperborea (L.) R.Br. ex Ait.f.] NORTHERN GREEN BOG ORCHID.

PLANTAGINACEAE (Plantain Family):

-Plantago eriopoda Torr. - SALINE or ALKALI PLANTAIN.

-Plantago major L. * - COMMON PLANTAIN.

POACEAE (=GRAMINEAE) (Grass Family):

-Achnatherum hymenoides (R.\& S.) Barkw. - INDIAN RICE-GRASS. [Oryzopsis hymenoides (R.\& S.) Rich. ex Pip.].

[X Agrohordeum macounii ( $=X$ Elyhordeum macounii)].

[Agropyron albicans (=Elymus albicans)].

-Agropyron cristatum (L.) Gaertn. ssp. pectinatum (Bieb.) Tzv. * [Agropyron - 
pectiniforme R.\& S.] - CRESTED WHEATGRASS.

[Agropyron dasystachyum var. dasystachyum (=Elymus lanceolatus var. lanceolatus).

[Agropyron intermedium (=Thinopyrum intermedium)].

[Agropyron repens (=Elymus repens)].

[Agropyron smithii (=Pascopyrum smithii)].

[Agropyron subsecundum (=Elymus trachycaulus ssp. subsecundus)].

[Agropyron trachycaulum ssp. trachycaulum (=Elymus trachycaulus ssp.

trachycaulus)].

[Agropyron riparium (=Elymus lanceolatus var. riparius)].

-Agrostis scabra Willd. - ROUGH BENT-GRASS.

-Agrostis stolonifera L. - REDTOP; CREEPING BENT.

[Andropogon scoparius (=Schizachyrium scoparium)].

-Bouteloua gracilis (Willd. ex Kunth) Lag. ex Griffiths - BLUE GRAMA-GRASS.

-Bromus ciliatus L. - FRINGED BROME.

-Bromus inermis Leyss. ssp. inermis * - SMOOTH BROME.

-Bromus tectorum L. * - DOWNY CHESS or BROME.

-Calamagrostis canadensis (Michx.) Beauv. - BLUEJOINT; MARSH REED-

GRASS.

-Calamagrostis montanensis Scribn. ex Vasey - PLAINS REED-GRASS.

-Calamagrostis stricta (Timm) Koel. spp. inexpansa (Gray) C.W.Greene [C. inexpansa Gray] - NORTHERN REED-GRASS.

-Calamagrostis stricta (Timm) Koel. ssp. stricta [C. neglecta (Ehrh.) Gaertn., Mey.\& Scherb.] - NARROW REED-GRASS.

-Calamovilfa longifolia (Hook.) Scribn. - SAND-GRASS.

- Catabrosa aquatica (L.) Beauv. - BROOK-GRASS.

- Deschampsia caespitosa (L.) Beauv. - TUFTED HAIR-GRASS.

-Distichlis spicata (L.) Greene var. stricta (Torr.) Beetle [D. stricta (Torr.) Rydb.] INLAND SALT GRASS.

-Echinochloa crusgalli (L.) Greene var. crusgalli * - BARNYARD GRASS.

$X$ Elyhordeum macounii (Vasey) Barkw. \& Dewey [=Elymus trachycaulus $X$

Hordeum jubatum; X Agrohordeum macounii Vasey]. - MACOUN'S WILD-RYE. -Elymus albicans (Scribn.\& Sm.) Dewey [Agropyron albicans Scribn.\& Sm.] AWNED NORTHERN WHEAT-GRASS.

-Elymus canadensis L. var. canadensis - CANADA or NODDING WILD-RYE.

[Elymus junceus (=Psathyrostachys juncea)].

-Elymus lanceolatus (Scribn.\& Sm.) Gould var. lanceolatus [Agropyron dasystachyum (Hook.) Scribn.\& Sm. var. dasystachyum] - NORTHERN WHEATGRASS.

-Elymus lanceolatus (Scribn.\& Sm.) Gould var. riparius (Scribn. \& Sm.) Dorn [Agropyrum riparium Scribn.\& Sm.; A. dasystachyum (Hook.) Scribn.\& Sm. var. riparium (Scribn.\& Sm.) Bowden] — STREAMBANK WHEATGRASS.

-Elymus repens (L.) Gould * [Agropyron repens (L.) Beauv.] - QUACK-GRASS; COUCH-GRASS.

-Elymus trachycaulus (Link) Gould \& Shin. ssp. subsecundus (Link) A.\& D.Love [Agropyron subsecundum (Link) A.S. Hitchc.; A. trachycaulum (Link) Malte ex Lewis var. unilaterale (Cass.) Moore] - AWNED WHEATGRASS.

-Elymus trachycaulus (Link) Gould \& Shin. ssp. trachycaulus [Agropyron trachycaulum (Link) Malte ex Lewis] - SLENDER or WESTERN WHEATGRASS. 
-Festuca altaica Trin. ssp. hallii (Vasey) Harms [F. hallii (Vasey) Piper; F. scabrella auct. non Torr.] - PLAINS ROUGH FESCUE.

- Festuca saximontana Rydb. var. saximontana [F. ovina $L$. var. saximontana (Rydb.) Gl.; F. ovina auct. non L.] - ROCKY MOUNTAIN FESCUE. -Glyceria striata (Lam.) A.S. Hitchc.- FOWL MANNA-GRASS. -Helictotrichon hookeri (Scribn.) Henr. - HOOKER'S OATGRASS. - Hesperostipa comata (Trin. \& Rupr.) Barkw. [Stipa comata Trin.\& Rupr.] NEEDLE-AND-THREAD SPEARGRASS; NEEDLE GRASS.

- Hesperostipa curtiseta (A.S. Hitchc.) Barkw. [Stipa curtiseta (A.S. Hitchc.) Barkw. ; S. spartea Trin. var. curtiseta A.S. Hitchc.] - WESTERN or SHORTAWNED NEEDLE-GRASS or PORCUPINE GRASS.

-Hierochloe hirta (Schrank) Borbas ssp. arctica (J.Presl.) Weim. [H. odorata auct. non (L.) Beauv.] - NORTHERN SWEET GRASS.

-Hordeum jubatum L. ssp. intermedium Bowden [H. jubatum L. var. caespitosum (Scribn. ex Pam.) A.S.Hitchc.] - SHORT-AWNED WILD BARLEY.

-Hordeum jubatum L. ssp. jubatum - FOXTAIL or WILD BARLEY.

-Koeleria macrantha (Ledeb.) Schultes [K. cristata auct. non Pers.] — JUNE GRASS.

-Lolium perenne L. ${ }^{*}$ - PERENNIAL or ENGLISH RYE-GRASS.

- Muhlenbergia cuspidata (Torr. ex Hook.) Rydb. - PRAIRIE MUHLY.

- Muhlenbergia racemosa (Michx.) BSP. - MARSH MUHLY.

- Muhlenbergia richardsonis (Trin.) Rydb. - MAT MUHLY.

- Nassella viridula (Trin.) Barkw. [Stipa viridula Trin.] - GREEN NEEDLE-GRASS.

-Oryzopsis asperifolia Michx. - WHITE-GRAINED MOUNTAIN-RICE.

[Oryzopsis hymenoides (=Achnatherum hymenoides)].

-Pascopyrum smithii (Rydb.) A.Love - WESTERN WHEAT-GRASS. [Agropyron smithii Rydb.].

-Phalaris arundinacea L. - REED CANARY-GRASS.

-Poa arida Vasey - PLAINS or PRAIRIE BLUEGRASS.

-Poa compressa L. * - CANADA or FLAT-STEMMED BLUE-GRASS.

-Poa cusickii Vasey - EARLY or CUSICK BLUEGRASS.

-Poa palustris L. - FOWL BLUE-GRASS.

-Poa pratensis L. * - KENTUCKY BLUEGRASS.

-Poa secunda PresI. [P. sandbergii Vasey] - CURLY, ALKALI, CANBY'S or SANDBERG'S BLUE-GRASS.

-Psathyrostachys juncea (Fisch.) Nevski * [Elymus junceus Fisch.] - RUSSIAN WILD-RYE.

-Puccinellia nuttalliana (Schultes) A.S. Hitchc. - NUTTALL'S ALKALI-GRASS.

-Schizachne purpurascens (Torr.) Swall. - PURPLE OAT-GRASS.

-Schizachyrium scoparium (Michx.) Nash ssp. scoparium [Andropogon

scoparius Michx.] - LITTLE BLUESTEM.

-Spartina gracilis Trin.- ALKALI CORDGRASS.

-Sphenopholis intermedia (Rydb.) Rydb. - SLENDER WEDGE-GRASS.

-Sporobolus cryptandrus (Torr.) Gray - SAND DROPSEED.

[Stipa comata (=Hesperostipa comata)].

[Stipa curtiseta (=Hesperostipa curtiseta)].

[Stipa viridula (=Nassella viridula)].

-Thinopyrum intermedium (Host) Barkw. \& Dewey * [Agropyron intermedium

(Host) Beauv.] - INTERMEDIATE WHEAT-GRASS.

-Triticum aestivum L. * - WHEAT. 
POLEMONIACEAE (Phlox Family):

-Collomia linearis Nutt. - NARROW-LEAVED COLLOMIA.

-Phlox hoodii Richards. - MOSS PHLOX.

\section{POLYGONACEAE (Buckwheat Family):}

- Eriogonum flavum Nutt. - YELLOW UMBRELLA-PLANT.

-Polygonum achoreum Blake * [P. erectum auct. non L.] - STRIATE or LEATHERY KNOTWEED.

-Polygonum arenastrum Jord. ex Boreau. * [P. aviculare auct. non L.] COMMON or YARD KNOT-WEED; DOORWEED.

-Polygonum amphibium L. var. stipulaceum Coleman - WATER or SWAMP PERSICARIA or SMARTWEED.

-Polygonum coccineum Muhl. ex Willd. var. pratincola (Greene) Stanford SWAMP SMARTWEED or PERSICARIA.

-Polygonum convolvulus L. * - WILD BUCKWHEAT; BLACK BINDWEED.

-Polygonum lapathifolium L. - WILLOW-LEAVED PERSICARIA or SMARTWEED.

-Rheum rhabarbarum L. * [R. rhaponticum L.] - RHUBARB.

-Rumex aquaticus L. var. fenestratus (Greene) Dorn [R. occidentalis S.Wats.] WESTERN DOCK.

-Rumex maritimus L. ssp. fueginus (Phil.) Hultén - GOLDEN DOCK.

-Rumex pseudonatronatus Borbas * [R. fennicus Murb.] - FIELD DOCK.

-Rumex stenophyllus Ledeb. ${ }^{*}$ - NARROW-LEAVED FIELD DOCK.

POTAMOGETONACEAE (Pondweed Family):

-Potamogeton crispus L. * - CURLY PONDWEED.

[Potamogeton filiformis (=Stuckenia filiformis)].

-Potamogeton foliosus Raf. ssp. foliosus - LEAFY PONDWEED.

[Potamogeton pectinatus (=Stuckenia pectinatus)].

-Stuckenia filiformis (Pers.) Boerner [Potamogeton filiformis Pers.] - THREADLEAVED PONDWEED.

-Stuckenia pectinata (L.) Boerner [Potamogeton pectinatus ] - SAGO or

FENNEL-LEAVED PONDWEED.

\section{PRIMULACEAE (Primrose Family):}

-Androsace septentrionalis L. ssp. septentrionalis - NORTHERN PIGMY-

FLOWER; FAIRY CANDELABRUM.

-Dodecatheon pulchellum (Raf.) Merr. [D. pauciflorum Greene] - BEAUTIFUL or SALINE SHOOTINGSTAR.

- Glaux maritima L. - SEA MILKWORT.

-Lysimachia ciliata L. - FRINGED YELLOW LOOSESTRIFE

PYROLACEAE (Wintergreen Family):

-Orthilia secunda (L.) House [Pyrola secunda L.] - ONE-SIDED

WINTERGREEN or PYROLA.

\section{RANUNCULACEAE (Buttercup Family):}

- Actaea rubra (Ait.) Willd. - BANEBERRY.

-Anemone canadensis L. - CANADA or MEADOW ANEMONE.

-Anemone cylindrica Gray - CANDLE, LONG-HEADED or LONG-FRUITED 
ANEMONE.

-Anemone multifida Poir. - CUT-LEAVED ANEMONE.

[Anemone patens (=Pulsatilla patens)].

-Pulsatilla patens (L.) P.Mill. ssp. multifida (Pritz.) Zamels [P. ludoviciana Hell.; -Anemone patens $L$. var. wolfgangiana (Bess.) Koch] - PRAIRIE CROCUS;

PASQUE-FLOWER; CROCUS ANEMONE.

-Ranunculus cymbalaria Pursh - SHORE, SEASIDE or ALKALI BUTTERCUP. -Ranunculus sceleratus L. - CELERY-LEAVED or CURSED BUTTERCUP.

- Thalictrum dasycarpum Fisch. \& Ave. - PURPLE or TALL MEADOW-RUE.

-Thalictrum venulosum Trel. - VEINY or EARLY MEADOW-RUE.

\section{RHAMNACEAE (Buckthorn Family):}

-Rhamnus alnifolia L'Her. - ALDER-LEAVED BUCKTHORN.

-Rhamnus cathartica L. ${ }^{*}$ - EUROPEAN BUCKTHORN.

\section{ROSACEAE (Rose Family):}

-Amelanchier alnifolia Nutt. - SASKATOON-BERRY; SERVICE-BERRY. -Argentina anserina (L.) Rydb. [Potentilla anserina L.] - SILVERLEAF; SILVERWEED; SILVER-FEATHER.

-Chamaerhodos erecta (L.) Bunge ssp. nuttallii (Pick. ex Rydb.) Hult. AMERICAN CHAMAERHODOS; LITTLE GROUND-ROSE.

-Crataegus chrysocarpa Ashe [C. rotundifolia auct. non Moench] - ROUNDLEAVED or FIREBERRY HAWTHORN.

-Fragaria vesca L. ssp. americana (Porter) Staudt - AMERICAN WILD or WOOD STRAWBERRY. [F. americana (Porter) Britt.].

-Fragaria virginiana Duch. ssp. glauca (S.Wats.) Staudt - SMOOTH WILD STRAWBERRY.

- Geum aleppicum Jacq. - YELLOW AVENS.

-Geum triflorum Pursh - THREE-FLOWERED AVENS; PRAIRIE SMOKE; OLDMAN'S-WHISKERS.

-Pentaphylloides floribunda (Pursh) A.Love [Potentilla fruticosa auct. non L.] SHRUBBY CINQUEFOIL.

[Potentilla anserina (=Argentina anserina)].

-Potentilla arguta Pursh - WHITE or TALL CINQUEFOIL.

-Potentilla bipinnatifida Dougl. ex Hook. - PLAINS CINQUEFOIL

[Potentilla fruticosa (=Pentaphylloides floribunda)].

-Potentilla gracilis Dougl. ex Hook. - GRACEFUL CINQUEFOIL.

-Potentilla hippiana Lehm. - WOOLLY or BRANCHED CINQUEFOIL.

-Potentilla pensylvanica L. - PRAIRIE CINQUEFOIL.

-Prunus pensylvanica L. f. var. pensylvanica - PIN, BIRD or FIRE CHERRY. -Prunus virginiana L. var. virginiana - COMMON or RED-FRUITED CHOKE CHERRY.

-Prunus virginiana L. var. melanocarpa (A.Nels.) Sarg. - BLACK-FRUITED CHOKE-CHERRY.

-Rosa acicularis Lindl. ssp. sayi (Schwein.) W.H. Lewis - PRICKLY WILD ROSE.

-Rosa arkansana Porter - LOW PRAIRIE ROSE.

-Rosa woodsii Lindl. - WOOD'S WILD ROSE.

-Rubus idaeus L. ssp. strigosus (Michx.) Focke - WILD, WESTERN, CANADIAN or AMERICAN RED RASPBERRY. 
Sorbus aucuparia L. * - EUROPEAN MOUNTAIN-ASH; ROWAN-TREE. -Spiraea alba Du Roi - WHITE or NARROW-LEAVED MEADOWSWEET.

RUBIACEAE (Madder Family):

- Galium boreale L. - NORTHERN BEDSTRAW.

-Galium triflorum Michx. - SWEET-SCENTED or FRAGRANT BEDSTRAW.

\section{SALICACEAE (Willow Family):}

-Populus balsamifera L. - BALSAM or BLACK POPLAR.

-Populus deltoides Bartr. ex Marsh. ssp. monilifera (Ait.) Eckenw. [P. d. var. occidentalis Rydb.; P. sargentii Dode] - WESTERN or PLAINS COTTONWOOD. -Populus tremuloides Michx. - TREMBLING ASPEN; WHITE POPLAR. -Salix bebbiana Sarg. - BEAKED or GRAY WILLOW.

-Salix candida Fluegge ex Willd. - HOARY or SAGE WILLOW.

-Salix discolor Muhl. - PUSSY WILLOW.

-Salix eriocephala Michx. var. famelica Ball [S. lutea auct. non Nutt.] YELLOW WILLOW.

-Salix exigua Nutt. ssp. interior (Rowlee) Cronq. - SANDBAR, COYOTE, or NARROW-LEAVED WILLOW.

-Salix petiolaris Sm. - MEADOW or BASKET WILLOW.

-Salix planifolia Pursh - FLAT-LEAF WILLOW.

-Salix pseudomonticola Ball [S. monticola auct. non Bebb.] - FALSE

MOUNTAIN or CHERRY-LEAVED WILLOW.

\section{SANTALACEAE (Sandalwood Family):}

-Comandra umbellata (L.) Nutt. ssp. umbellata - COMMON COMANDRA; BASTARD TOADFLAX.

-Comandra umbellata (L.) Nutt. ssp. pallida (DC.) Piehl - PALE or WESTERN COMANDRA; BASTARD TOADFLAX.

\section{SAXIFRAGACEAE (Saxifrage Family):}

- Heuchera richardsonii R.Br. - RICHARDSON'S ALUMROOT.

-Parnassia palustris L. var. tenuis Wahl. - MEADOW, NORTHERN, or MARSH GRASS-OF-PARNASSUS.

SCROPHULARIACEAE (Figwort Family):

-Orthocarpus luteus Nutt. - OWL'S-CLOVER.

-Penstmon gracilis Nutt. - LILAC BEARDTONGUE.

-Penstemon nitidus Dougl. ex Benth.- SMOOTH BLUE BEARDTONGUE.

-Veronica scutellata L. - MARSH or GRASS-LEAVED SPEEDWELL.

SELAGINELLACEAE (Little Clubmoss Family):

-Selaginella densa Rydb. - PRAIRIE SELAGINELLA or SPIKE-MOSS.

SMILACACEAE (Carrion-flower Family):

-Smilax lasioneuron Hook. - CARRION-FLOWER.

SOLANACEAE (Nightshade Family):

-Solanum triflorum Nutt. - WILD TOMATO. 
TYPHACEAE (Cattail Family):

-Typha latifolia L. - COMMON or BROAD-LEAVED CATTAIL.

ULMACEAE (Elm Family):

- UImus americana L. * - AMERICAN or WHITE ELM.

-UImus pumila L. * - MANCHURIAN or SIBERIAN ELM.

URTICACEAE (Nettle Family):

- Urtica dioica L. var. gracilis (Ait.) Seland. [U. procera Muhl. ex Willd.; U. Iyallii S.Wats.] - COMMON STINGING NETTLE.

\section{VIOLACEAE (Violet Family):}

- Viola adunca Sm. - EARLY BLUE or HOOKED-SPUR VIOLET.

- Viola canadensis L. var. rugulosa (Greene) C.L. Hitchc. [V. rugulosa Greene]

- WESTERN CANADA WHITE VIOLET; TALL WHITE VIOLET.

- Viola nephrophylla Greene - NORTHERN BOG VIOLET.

- Viola vallicola A.Nels. [V. nuttallii Pursh var. vallicola (A.Nels.) St.John] -

YELLOW PRAIRIE or VALLEY VIOLET.

ZANNICHELLIACEAE (Horned-Pondweed Family):

-Zannichellia palustris L. - HORNED-PONDWEED.

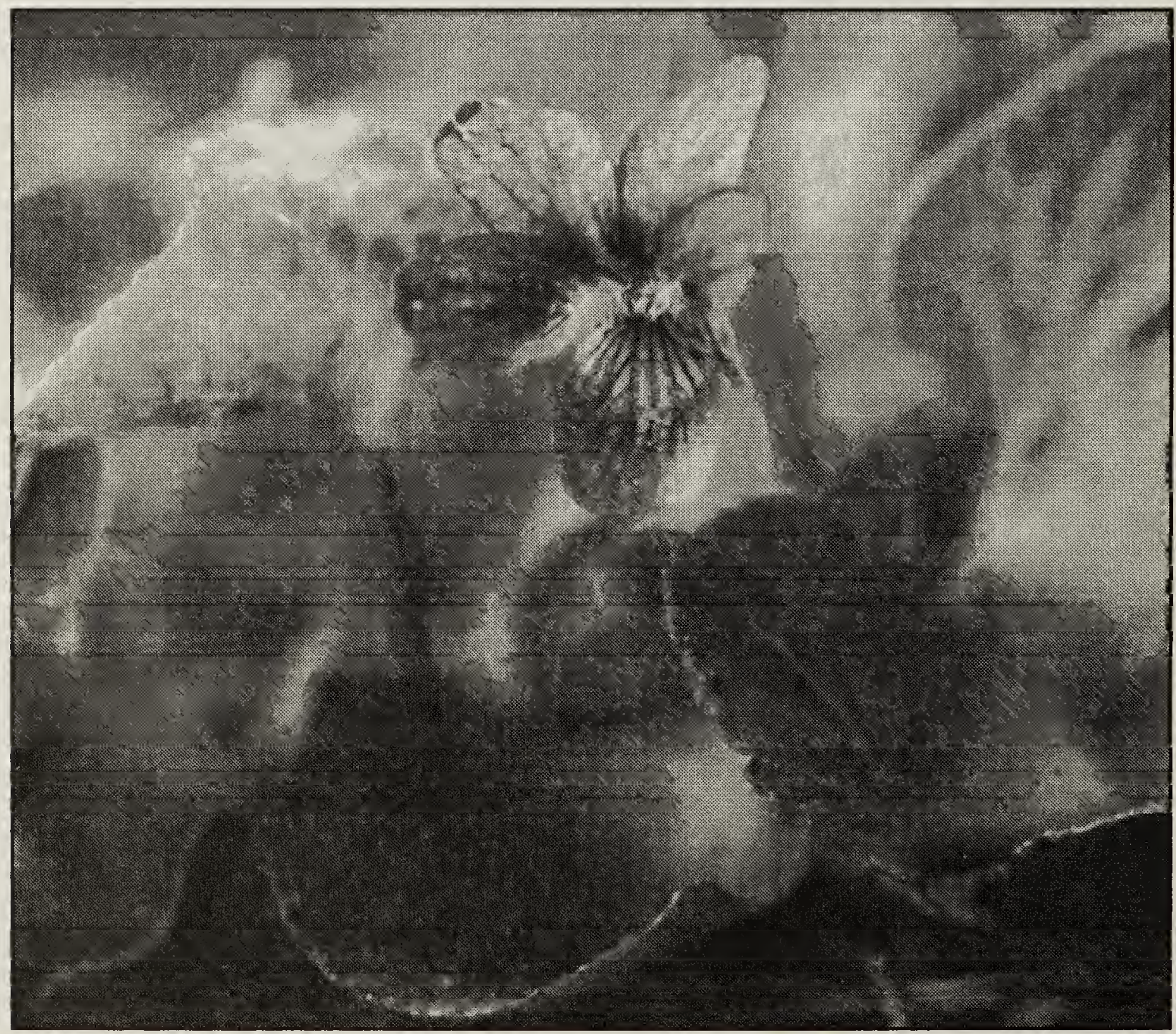


1. Delanoy, L. (1997?). Vegetation Survey of Natural Riverbank Lands North and West of the Regional Psychiatric Centre. Internal Document. Meewasin Valley Authority, Saskatoon, SK.

2. Fraser, W. P., and R. C. Russell. 1944. A Revised, Annotated List of the Plants of Saskatchewan. University of Saskatchewan, Saskatoon, SK.

3. Golder Associates Ltd., et al 1995. Peturrson's Ravine Management Plan. Report prepared for Meewasin Valley Authority.

4. Harms, V. L. 2000. Rare Native Vascular Plants of Saskatchewan. W.P. Fraser Herbarium, University of Saskatchewan, Saskatoon, SK.
5. Hudson, J. H. 1951-1987. Field journal notes (Saskatoon area abstracted). Unpublished.

6. Kartesz, J.T. 1999. A synonymized checklist and atlas with biological attributes for the vascular flora of the United States, Canada, and Greenland. First Edition. In: Kartesz, J.T., and C.A. Meacham (Eds.). Synthesis of North American Flora. Version 1.0. North Carolina Botanical Garden, Chapel Hill, NC.

7. Lineman, M. J. 1993. A Botanical Inventory of Some Natural Areas in the South Saskatchewan River Valley in and near Saskatoon. Part II - 1993 Site Surveys (Site 12). Report for Saskatoon Natural History Society and the Meewasin Valley Authority. Nov. 1993. 40 pp +126 pp. appendices.

\section{PEZIZA PROTEANA VAR. SPARASSOIDES, AN UNUSUAL FUNGUS FOUND ON A FARM IN FISKE, SK}

SUSAN KAMINSKYJ, Department of Biology, University of Saskatchewan, 112 Science Place, Saskatoon, SK S7H 2Z7. Email: Susan.Kaminskyj@usask.ca

In October 2000, Don Graham, who farms near Fiske, Saskatchewan, brought me a cream-coloured specimen of a fungus that looked superficially like a boiled cabbage (Figure 1). The sample weighed more than a kilogram, and had been broken off a much larger mass, probably at least four kilograms. It was not immediately clear what it was, but fortunately Don's wife Shirley had thought to freeze it - this is the best way to store fungal specimens until they can be preserved - and I was able to examine a piece under a microscope. It turned out to be the fruiting body of a type of ascomycete (a group that bears its spores in sacs called asci; Figure 2).

After discussing Don's find with experts in Edmonton, Prince George $\mathrm{BC}$, and Georgia (USA), the fungus was identified as Peziza proteana variety 